\title{
Inertial sensing with quantum gases: a comparative performance study of condensed versus thermal sources for atom interferometry
}

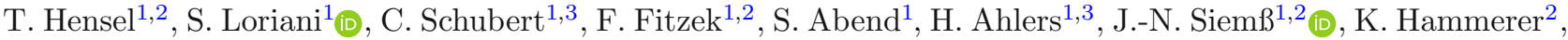 \\ E. M. Rasel ${ }^{1}$, and N. Gaaloul ${ }^{1, a}(\mathbb{D}$ \\ 1 Institute of Quantum Optics, Leibniz University Hannover, Welfengarten 1, 30167 Hannover, Germany \\ 2 Institute for Theoretical Physics, Leibniz University Hannover, Appelstraße 2, 30167 Hannover, Germany \\ 3 Institut für Satellitengeodäsie und Inertialsensorik, c/o Leibniz Universität Hannover, DLR-SI, Callinstraße 36, 30167 \\ Hannover, Germany
}

Received 15 September 2020 / Accepted 2 February 2021

(C) The Author(s) 2021, corrected publication 2021

\begin{abstract}
Quantum sensors based on light pulse atom interferometers allow for measurements of inertial and electromagnetic forces such as the accurate determination of fundamental constants as the fine structure constant or testing foundational laws of modern physics as the equivalence principle. These schemes unfold their full performance when large interrogation times and/or large momentum transfer can be implemented. In this article, we demonstrate how interferometry can benefit from the use of Bose-Einstein condensed sources when the state of the art is challenged. We contrast systematic and statistical effects induced by Bose-Einstein condensed sources with thermal sources in three exemplary science cases of Earth- and space-based sensors.
\end{abstract}

\section{Introduction}

Atom interferometers are mainly used for inertially sensitive measurements [1] and a variety of tests of fundamental physics $[2,3]$. Key levers to increase the sensitivity are the transfer of a large number of photons during the beam-splitting processes, extending the time of free fall while maintaining contrast and atomic flux. At the same time, the characterization of errors requires an increased level of control over the manipulation and preparation of atoms. Limitations of interferometers operating with molasses-cooled atoms and their mitigation by reducing the residual expansion rates were studied theoretically $[4]$ and experimentally $[5,6]$.

In this paper, together with the trade-off between flux and expansion rate, we contrast the appropriateness of the two regimes of atomic ensembles to perform tests with high accuracy and stability. To this end, we evaluate respective contributions to statistical uncertainties such as shot noise, cycle times and mean-field interactions, and the most prominent systematics such as gravity gradients (GGs), Coriolis force and wave front aberrations (WFA).

Proposals for space missions, in particular, rely on Delta-Kick collimation (DKC) via optical or magnetic potentials to exploit extended times of free fall in microgravity $[7-10]$ and achieve extremely low wave packet

\footnotetext{
a e-mail: gaaloul@iqo.uni-hannover.de (corresponding author)
}

expansion rates, corresponding to $\mathrm{pK}$ temperatures in thermal ensembles. Bose-Einstein condensed (BEC) ensembles are better suited for DKC aiming at long interrogation times [4], but suffer from a reduced atomic flux due to the evaporation despite recent promising studies [11]. Molasses-cooled atoms feature a higher number of atoms, but are typically velocity-filtered in 1D [12], which ultimately implies a lower flux of atoms as we will detail in our study.

To illustrate our comparative study between condensed and thermal sources, we consider three prominent cases for free fall atom interferometers: a gravimeter, a gravity-gradiometer, and a test of the universality of free fall (also known as Weak Equivalence Principle (WEP) test). Thermal sources are defined, in this study, as atomic ensembles with a vanishing condensed fraction. Conversely, BEC sources possess a condensed fraction of $100 \%$. For each case, we limit the maximum allowed diameter of the atomic ensemble at the recombination pulse to preserve a high diffraction efficiency for all beam-splitting operations. Subsequently, this enables the determination of various measurement uncertainty contributions for the trade-off.

This article is structured as follows. Starting with a brief overview of the state of the art of light pulse atom interferometry (Sect. 1.1), we continue with relevant uncertainty contributions to the read-out phases of atom interferometers (Sect. 2), quantitatively evaluating them in the three study cases (Sect. 3) andfinally - discussing the limits of the condensed or ther- 
mal regime of the respective interferometry source (Sect. 4).

\subsection{State of the art}

Experiments based on the interference of freely falling atoms measure accelerations [1,13], rotations [14-18], gravity gradients $[19,20]$, determine fundamental constants $[2,21,22]$, perform tests of fundamental physics $[2,3,22-27]$, and are proposed for the detection of gravitational waves [4,28-31]. A recent review of the advances in the field of inertial sensing collects most relevant experiments and proposals so far [1,32]. Beyond proof-of-principle demonstrations, ongoing developments target commercialization as well as challenge the state of the art in sensor performance and in fundamental science [33].

Prominent examples of atomic inertial sensors with molasses cooled atoms are gravimeters $[34,35]$ reaching a sensitivity of $4 \times 10^{-8}\left(\mathrm{~m} / \mathrm{s}^{2}\right) / \sqrt{\mathrm{Hz}}[36]$ and an uncertainty of $3 \times 10^{-8} \mathrm{~m} / \mathrm{s}^{2}[37,38]$ as well as gyroscopes $[18,39,40]$ with a sensitivity of $3 \times 10^{-8}(\mathrm{rad} / \mathrm{s}) / \sqrt{\mathrm{Hz}}$ [41]. BEC gravimeters $[42,43]$ offer the perspective of a lowered uncertainty $[5,44]$ in compact $[45]$ and transportable setups [46]. In a large fountain, a BEC interferometer demonstrated an intrinsic noise corresponding to a sensitivity to accelerations of $3 \times 10^{-10}\left(\mathrm{~m} / \mathrm{s}^{2}\right) / \sqrt{\mathrm{Hz}}$ [47].

With atom interferometry, the gravitational constant $G$ is determined to a value $G=6.67191(99) \times$ $10^{-11} \mathrm{~m}^{3} \mathrm{~kg}^{-1} \mathrm{~s}^{-2}$ with a relative uncertainty of $150 \mathrm{ppm}$ [22]. The precision is limited by the spatial (vertical) spread of the cloud at the beginning of the sequence.

Most recently $[21,48,49]$, there has been extensive work on the determination of the fine-structure constant $\alpha$ via determination of the ratio $\hbar / m$ with matterwave interferometry, where $m$ is the atomic mass and $\hbar$ is Planck's reduced constant. In a fountain with thermal cesium atoms, the fine-structure constant is determined with an expected statistical error of $0.008 \mathrm{ppb}$ [48]. The systematic uncertainty is at the $0.12 \mathrm{ppb}$ level, mainly stemming from spurious accelerations. With thermal rubidium, $\hbar / m$ is measured at the $4.5 \times 10^{-9}$ level [50]. An ytterbium contrast interferometer with BECs [51] is used to demonstrate an $\hbar / m$-measurement using large momentum transfer, controlling diffraction effects and atomic interactions with suppression of vibrational effects allowing sub-ppb relative measurement uncertainty.

In [26], a dual-species WEP test with ${ }^{85} \mathrm{Rb}$ and ${ }^{87} \mathrm{Rb}$ reaches a statistical uncertainty of $\eta=0.8 \times 10^{-8}$ and is limited by systematic effects, e.g., the Coriolis effect to $\eta=(2.8 \pm 3.0) \times 10^{-8}$. Most recently, this limit has been pushed further down to $\eta=1.6 \pm 5.2 \times 10^{-12}[3]$. The STE-QUEST mission [52] aims at testing the WEP at the $10^{-15}$ level and beyond by measuring the differential acceleration of a ${ }^{87} \mathrm{Rb} \mathrm{BEC}$ and ${ }^{41} \mathrm{~K}$ BEC over a total mission time of 5 years [53]. The concept Quantum Test of the Equivalence principle and Space Time (QTEST) [54] intends to determine $\eta$ at the $10^{-15}$ level with thermal ensembles over four integration periods of three months each aboard the International Space Station.

\subsection{Performance indicators}

In the previous subsection, the state-of-the-art limits for measurements of rotations, accelerations, the fine-structure constant and the Eötvös ratio have been stated. The phase that is to be determined depends on several experimental parameters like the effective wave vector $k_{\text {eff }}$, the interrogation time $2 \mathrm{~T}$, the velocity $v$ of the atomic ensemble perpendicular to the sensitive axis, the length of the detector baseline $D$ and the frequency $f$ of the gravitational wave. For the commonly proposed interferometry schemes discussed above, one can summarize the performance-de-fining scaling factors to be:

- $k_{\text {eff }} T^{2}$ for gravimetry and WEP tests,

- $k_{\text {eff }}^{2} T$ for $\hbar / m$ measurements,

- $k_{\text {eff }} D \cos (f T)$ for gravitational wave detection,

- $k_{\text {eff }} D T^{2}$ for gravity gradiometry and $G$ measurements,

$-k_{\text {eff }} T^{2} v$ for rotations.

These scaling factors describe how the to-be-measured quantities (acceleration, rotation,...) are translated into a phase, and increasing them allows for an improved measurement sensitivity.

In general, various effects $S$ (gravity gradients, rotations, light wave front distortions) couple to the atomic source characteristics $q$ (initial position and velocity, density), leading to additional phase shifts. In case of a gravimeter, for example,

$$
\phi_{S}=k_{\mathrm{eff}} T^{2} \times S q,
$$

and a potential phase contribution $\phi_{\gamma}=k_{\mathrm{eff}} T^{2} \gamma z_{0}$ is given by gravity gradients $S=\gamma$ that couple to the initial position $q=z_{0}$ of an atom at the first pulse of the interferometric sequence. In the assessment of the sensor performance, the influence of the uncertainties $\delta S, \delta q$ on the phase is evaluated by quantifying the derived uncertainties $\delta \phi_{S}=S \delta q, q \delta S$ and $\delta S \delta q$.

The uncertainties can be of different origin, and in the following we will continuously use $\delta$ to denote systematic (bias) uncertainties and $\sigma$ for statistical uncertainties. As a concrete example: $\delta_{\phi, \gamma}=k_{\text {eff }} T^{2} z_{0} \delta_{\gamma}$ and $k_{\text {eff }} T^{2} \gamma \delta_{z_{0}}$ are systematic phase uncertainties due to limited knowledge about the values of $\gamma$ and $z_{0}$, whereas $\sigma_{\phi, \gamma}=k_{\text {eff }} T^{2} \gamma \sigma_{z_{0}}$ is a statistical uncertainty, given by the statistical distribution of the atomic position around the mean $z_{0}$, quantified by the standard deviation $\sigma_{z_{0}}$. The important difference is that the systematic contributions have to be controlled at the target accuracy level, which can be achieved through pre-interferometry characterization measurements (e.g., accurate determination of the mean position reduces $\delta_{z}$ ) or by minimizing the coupling factor 
$S$ (e.g., through compensation schemes [55]). The statistical uncertainty, on the other hand, is reduced by repeated measurements, i.e., realizations of the atom interferometer.

The figure of merit here is the intrinsic statistical shot noise due the measurement scheme. It limits the single-shot phase uncertainty to

$$
\sigma_{\phi_{\mathrm{SN}}}=1 /\left(C \sqrt{N_{\mathrm{at}}}\right)
$$

defined by the number of interfering atoms $N_{\mathrm{at}}$ and interferometric contrast $C$. The experiments are repeated $\mathrm{n}_{\text {cycle }}$ times to average ('integrate') this uncertainty.

With the assumption of a shot noise-limited measurement, a fixed atom number and no reduction in contrast $C$, an increase in the scale factor by enhancing the free evolution time $T$ or the effective wave number $k_{\text {eff }}$ can increase the single-shot phase sensitivity. Interrogation times $2 \mathrm{~T}$ of several seconds were realized [47] with BECs and an extension to $10 \mathrm{~s}$ was proposed on space platforms [52]. The effective momentum transfer ranges from few $10 \mathrm{k}_{\text {eff }}$ for a single multi-photon pulse up to a few $100 \mathrm{~s}$ of $\mathrm{k}_{\text {eff }}[56]$ for benchmark experiments. The integration time to reach the desired performance may range from typically $10^{4} \mathrm{~s}$ up to several months. Generally, the large number of atoms and smaller cycle time in thermal ensembles is an advantage over BECs to reduce shot noise. Another statistical effect, the influence of mean-field is also expected to be suppressed efficiently through the lower density of thermal gases. On the other hand, their spatial extent limits the efficiency of atom optics operations (effectively resulting in increased statistical uncertainties due to atom loss) and leads to larger systematics. In the next section, these performance-limiting effects are discussed before being quantified in three study cases.

\section{Performance-limiting effects in atom interferometry}

Apart from shot noise considerations, a variety of physical phenomena limits the achievable accuracy and stability.

In the following, we characterize different systematic and statistical effects that might limit nearfuture experiments beyond state of the art such as long-fountain atomic gravimeters, space-based atom interferometers and atom interferometers operated in ground-based laboratories in micro-gravity environments. Starting with intrinsic loss mechanisms due to matter-light interaction, we go on to discuss different systematic (GGs, Coriolis effect, WFA) and statistical (mean-field) contributions to the uncertainty budget. After presenting DKC as a technique to suppress most of these effects, we conclude this section by analyzing the impact of imperfect detection of the atomic sample.

\subsection{Coherent manipulation}

The fidelity of the interferometric beam splitters and mirrors realized by the coherent manipulation of the atoms using light is closely connected to the phasespace properties of the atomic ensemble.

First, homogeneous excitation of the atomic ensemble requires a constant Rabi frequency over the spatial extent of the atoms, which in turn implies a laser beam size much larger than the ensemble size. In cases of optical power constraints, e.g., typical for space missions, the ensemble size is hence restricted in order to maintain contrast by achieving reasonable rates for coherent manipulation. For large free fall times such a requirement can be translated into a maximum expansion rate of the ensemble. Figure 1 shows the significant difference in expansion rate between thermal and condensed ensembles which indicates a clear advantage of the latter especially for large interferometry time scales. Second, for all applications outlined in the previous section, typically Doppler-sensitive two-photon couplings are employed, such that the longitudinal atomic velocity introduces a detuning. The temporal profile of light pulses determines their velocity acceptance and hence defines criteria for the atoms' velocity dispersion. In general, smaller velocity widths, corresponding to a smaller distribution of detunings, are desirable for an efficient addressing of the atoms.

An effective, simplified model [57] allows to assess the effects of spatial and velocity selectivity quantitatively. The convolution of the atoms' radial density distribution $n(\mathbf{r}, t)$ and longitudinal dispersion $f(\mathbf{v})$ with the position- and velocity-dependent excitation rate of the pulse

$$
p(\mathbf{r}, \mathbf{v}, t) \propto\left(\Omega_{0} / \Omega_{\mathrm{eff}}\right)^{2} \sin ^{2}\left(\Omega_{\mathrm{eff}} t / 2\right)
$$

determines the total excitation rate

$$
P_{\text {exc }}=2 \pi \iint \mathbf{r} f(\mathbf{v}) n(\mathbf{r}, t) p(\mathbf{r}, \mathbf{v}, t) \mathrm{d} \mathbf{r} \mathrm{d} \mathbf{v} .
$$

Note, that the transverse velocity distribution modifies the time-dependent spatial distribution $n(\mathbf{r}, t)$. This treatment assumes a box pulse of duration $t$ and an effective Rabi frequency

$$
\Omega_{\mathrm{eff}}=\sqrt{\Omega_{0}^{2}(\mathbf{r})+\left(\mathbf{k}_{\mathrm{eff}} \cdot \mathbf{v}\right)^{2}},
$$

where the spatial dependence of the Rabi frequency $\Omega_{0}(\mathbf{r})$ is given by the radial beam profile. A similar treatment was applied in the case of a recent gravitational wave detection proposal [4].

\subsection{Wave front aberrations}

Matter-light interactions in the atom-interferometric cycle are typically subject to the beam's natural wave front curvature (e.g., of a Gaussian beam) and additional imperfections of the laser beam profile $[5,6]$ caused by non-ideal optics. While errors due to the 
initial collimation of large beams $(>2 \mathrm{~cm})$ are negligible, retro-reflection still introduces WFA that lead to a considerable systematic uncertainty. We employ a second order approximation to the deviation from flat wave fronts for the combined effects of beam and optics. The resulting spatial dependence of the laser phase fronts imprints a position-dependent phase on the atoms. Depending on the amplitude and wavelength of the distortion relative to the size of the atomic ensemble, the resulting phase shift may average out, reduce contrast, lead to phase patterns that can be resolved during detection or result in an average phase shift. In state-of-the-art cold atom gravimeters, WFA induce the limiting systematic uncertainty of $30 \mathrm{~nm} / \mathrm{s}^{2}$ to $40 \mathrm{~nm} / \mathrm{s}^{2}$ $[37,38]$. A more recent analysis of the device in [37] evaluated the systematic bias to $55 \mathrm{~nm} / \mathrm{s}^{2}$ with an uncertainty of $13 \mathrm{~nm} / \mathrm{s}^{2}$ [44]. We limit our study case to longscale WFA, assuming a quadratic dependency of the wave fronts on the transverse position of the atoms, as introduced by a curvature of the retro-reflecting mirror. In this case, the resulting wave front curvature with radius $R$ couples to the finite velocity spread $\sigma_{v}$ and induces the phase shift

$$
\phi_{\mathrm{WFA}}=\frac{k_{\mathrm{eff}}}{R} \frac{k_{B} T_{\mathrm{at}}}{m_{\mathrm{at}}} T^{2}
$$

for a Mach-Zehnder (MZ) geometry, a spatial Gaussian density distribution of the ensemble and a Gaussian velocity spread $\sigma_{v}=\sqrt{k_{B} T_{\text {at }} / m_{\text {at }}}$ [5]. Here, $k_{\text {eff }}$ denotes the effective wave vector, $T$ the interrogation time, $k_{B}$ is the Boltzmann constant and $T_{\text {at }}$, $m_{\text {at }}$ refer to the ensemble temperature and atomic mass, respectively. Statistical fluctuations $\sigma_{T_{a t}}$ in the effective temperature lead to a phase noise $\sigma_{\phi_{\mathrm{WFA}}}=$ $k T^{2} k_{B} /\left(m_{\text {at }} R\right) \cdot \sigma_{T_{\text {at }}}$, whereas limited knowledge $\delta R$ about the wave front curvature results in a systematic uncertainty $\delta_{\phi_{\mathrm{WFA}}}=k T^{2} T_{\mathrm{at}} k_{B} /\left(m_{\mathrm{at}} R^{2}\right) \cdot \delta_{R}$.

\subsection{Mean-field effects}

Mean-field effects arise due to atom-atom interactions in atomic ensembles, scale with growing densities and are an additional source for statistical errors. The meanfield energy reads

$$
E_{\mathrm{MF}}(r)=g_{\text {int }} n(r)
$$

and depends on the local density $n(r)$ of the ensemble and the interaction strength $g_{\text {int }}^{\mathrm{BEC}}=4 \pi \hbar^{2} a_{s c} / m_{\text {at }}$, where $a_{s c}$ is the s-wave scattering length. For a thermal ensemble, $g_{\text {int }}^{\text {thermal }}=2 g_{\text {int }}^{\mathrm{BEC}}[58]$. Following [43], the average mean-field energy for a spherical ensemble of volume $V(t)=4 \pi r(t)^{3} / 3$ with $N_{\text {at }}$ atoms is consequently given by $\left\langle E_{\mathrm{MF}}\right\rangle=g_{\text {int }} N_{\text {at }} / V$. This assumes, however, a uniform density distribution with radius $r$ while thermal and BEC ensembles in fact follow a Gaussian or parabolic distribution, respectively. Hence, we take the average of the mean-field energy by weighting it with the respective density distribution:

$$
\begin{aligned}
\left\langle E_{\mathrm{MF}}\right\rangle & =\frac{4 \pi \int_{0}^{\infty} d r r^{2} n(r) E_{\mathrm{MF}}(r)}{4 \pi \int_{0}^{\infty} d r r^{2} n(r)} \\
& =g_{\mathrm{int}} \frac{\int_{0}^{\infty} d r r^{2} n^{2}(r)}{\int_{0}^{\infty} d r r^{2} n(r)} \\
\left\langle E_{\mathrm{MF}}^{\mathrm{BEC}}(t)\right\rangle & =\frac{15 g_{\mathrm{int}}^{\mathrm{BEC}} N_{\mathrm{at}}^{\mathrm{BEC}}}{14 \pi R_{\mathrm{TF}}^{3}(t)} \\
\left\langle E_{\mathrm{MF}}^{\text {thermal }}(t)\right\rangle & =\frac{g_{\text {int }}^{\text {thermal }} N_{\mathrm{at}}^{\text {thermal }}}{8 \pi^{3 / 2} \sigma_{r}^{3}(t)} .
\end{aligned}
$$

In case of an equal $g_{\text {int }}$, but unequal atomic density on the two arms of the atom interferometer, a spurious phase shift arises. Following [43], we model the contribution by linking the imbalance in density to the initial beam splitter and neglect effects due to overlap of the two arms or losses. If the initial beam splitter creates a superposition that deviates by $b$ from equal probability in both states, the phase shift

$$
\phi_{\mathrm{MF}}(t)=\frac{b}{\hbar} \int_{0}^{t}\left\langle E_{\mathrm{MF}}\left(r\left(t^{\prime}\right)\right)\right\rangle \mathrm{d} t^{\prime}
$$

occurs, corresponding to the integral of the differential frequency shift between the arms. This imbalance can be of systematic origin for a non-ideally configured initial beam splitter, which we assume to be characterized to the required level. In our assessment, we assume the initial superposition to have equal probabilities of both states on average, but to be affected by white noise with a standard deviation of $b=\sigma_{N}$ per cycle. Without relying on quantum correlations [59], it is given by quantum projection noise, implying an upper limit of $\sigma_{N}=1 / \sqrt{N_{\text {at }}}$ per cycle, which we adopt for our analysis.

\subsection{Gravity gradients and Coriolis effect}

Two of the most relevant effects are related to GGs and the Coriolis force. The first is the acceleration uncertainty due to the mass distribution of Earth and the apparatus surrounding the experiment. The second arises due to the transverse motion of the atoms with respect to the incident beam in combination with Earth's rotation, which forms an effective Sagnac interferometer [60,61]. Both give rise to additional phase shifts as they couple to the initial kinematic conditions of the ensemble [62].

In the case of a MZ geometry, the atom's initial velocity $v$ and position $r$ couple to GGs $\gamma$ parallel and perpendicular to the sensitive axis. The resulting phase is given by

$$
\begin{aligned}
\phi_{v, G G, \|} & =k_{\mathrm{eff}} \gamma_{\|} v T^{3} \\
\phi_{r, G G, \|} & =k_{\mathrm{eff}} \gamma_{\|} r T^{2} \\
\phi_{v, G G, \perp} & =\frac{14}{3} k_{\mathrm{eff}} \gamma_{\perp} v \Omega T^{4} \\
\phi_{r, G G, \perp} & =8 k_{\mathrm{eff}} \gamma_{\perp} r \Omega T^{3} .
\end{aligned}
$$


Similarly, residual rotations $\Omega$ perpendicular to the sensitive axis couple to the atom's velocity. The phase shift due to the Coriolis force (subscript $\mathrm{C}$ ) is given by

$$
\phi_{v, C, \perp}=2 k_{\mathrm{eff}} \Omega_{\perp} v T^{2} .
$$

Consequently, uncertainties in the initial kinematics directly translate into a phase uncertainty. As the phase-space properties of an atomic ensemble follow a statistical distribution, the spatial $\sigma_{r}$ and velocity $\sigma_{v}$ spread lead to phase noise per interferometric shot, given by $\sigma_{\phi, r, G G_{\perp}}=k_{\mathrm{eff}} \gamma_{\perp} \sigma_{r} T^{2}$ for example (analogous for the other terms). This constrains the possible width and effective expansion rate of the atomic source to keep those contributions below shot noise.

However, stricter requirements are typically demanded by the characterization of the mean initial position $\delta_{r}$ and velocity $\delta_{v}$ uncertainty to mitigate systematic shifts like $\delta_{\phi, r, G G_{\perp}}=k_{\text {eff }} \gamma_{\perp} \delta_{r} T^{2}$. The atomic ensemble that serves as input to the interferometer is imaged for the determination of the spatial properties, whereas timeof-flight measurements allow to quantify the velocity distribution. The accuracy of the determination of the mean position and velocity is governed by the statistics of the ensemble. Supposing the Gaussian density distribution of a thermal ensemble, they are related to the spatial $\sigma_{r}$ and velocity $\sigma_{v}$ spread [55], respectively, via

$$
\delta_{v, r}=\sigma_{v, r} / \sqrt{N_{\mathrm{at}} \nu_{0}}
$$

assuming a shot noise limited process. The number of atoms $N_{\text {at }}$ and the number of prerequisite measurements $\nu_{0}$ (i.e., the number of times the ensemble is imaged before the actual interferometric experiment to characterize the ensemble) equally contribute to the statistical repetition. BECs follow a parabolic density distribution. However, this can be approximated by a Gaussian distribution via Eq. 24, as explained in the next section.

Due to low order of magnitude of terms related to the coupling of rotations to transverse GGs, we restrict ourselves to phase uncertainties due to gradients parallel to the sensitive axis. Similarly, uncertainties due to the statistical distribution of the atoms around the mean position $r$ and systematics due to finite knowledge of the gravity gradient are comparably negligible in the following.

\subsection{Expansion rate and collimation}

Since expansion rates have sizable effects on the uncertainty of an atom-interferometric measurement, we discuss the possibilities offered by DKC to reduce them in this section. DKC is an established tool to further reduce the effective expansion rate of an ensemble (see [63] and references therein). This phase-space manipulation technique exploits that the free evolution of a gas released from a trap leads to a linear correlation of momentum and radial position of atoms within the ensemble. Subsequent trapping of the ensemble in a quadratic potential for a well-chosen duration leads to its collimation. Preservation of phase space density in the collisionless case requires that this reduction in momentum spread is accompanied by an increased ensemble size and hence necessitates a trade-off between desired expansion rate reduction and required growth in ensemble size. For non-interacting gases, i.e., thermal or fermionic gases in all regimes, this relation is captured by Liouville's theorem,

$$
\sigma_{v_{0}} / \sigma_{v_{f}}=\sigma_{r_{f}} / \sigma_{r_{0}}
$$

which states that the ratio of initial $\left(\sigma_{v_{0}}\right)$ to final $\left(\sigma_{v_{f}}\right)$ velocity width is inversely proportional to the relative increase in ensemble size $\left(\sigma_{r_{f}} / \sigma_{r_{0}}\right)$. A similar relation can be found for interacting degenerate gases - for which the asymptotic final expansion rate is determined by the initial localization through Heisenberg's uncertainty principle and a mean-field contribution $[4,64,65]$ - and for interacting, non-degenerate gases [58,66]. Figure 1 illustrates a DKC sequence collimating a BEC and a thermal ensemble with typical parameters. The free expansion of the thermal ensemble is governed by the expansion law

$$
\sigma_{r}(t)=\sqrt{\sigma_{r_{0}}^{2}+\sigma_{v_{f}}^{2} t^{2}}
$$

whereas the BEC dynamics are captured by corresponding scaling laws [64]. As an exemplary case, we take a thermal ensemble of $10^{9}{ }^{87} \mathrm{Rb}$ atoms at $2 \mu \mathrm{K}$ with a diameter of $2 \sigma_{r}=0.2 \mathrm{~mm}$ and collimate it down to $80 \mathrm{nK}$, such that the required size at lens is $2 \sigma_{r}\left(t_{\mathrm{DKC}}\right)=1 \mathrm{~mm}$. For a BEC, we assume an ensemble of $10^{6}{ }^{87} \mathrm{Rb}$ atoms and collimate it to $50 \mathrm{pK}$ in a trap with frequencies of $50 \times 2 \pi \mathrm{Hz}$. These are regimetypical parameters for experiments with either thermal ensembles or BECs $[4,9,10]$. Following [4], the expansion energies for the thermal atoms and the chemical

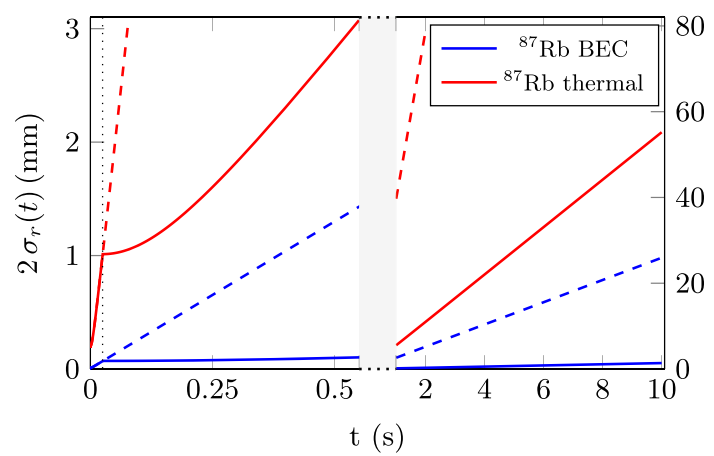

Fig. 1 Size evolution of thermal ensembles (red) and BECs (blue) after release from a trap. A DKC stage is reducing the expansion energies down to $80 \mathrm{nK}$ and $50 \mathrm{pK}$ for the thermal and BEC ensembles, respectively (see Table 1 for the exact parameters). The dashed lines illustrate the expansion in the freely expanding case without collimation 
Table 1 Parameters of the DKC sequence of ${ }^{87} \mathrm{Rb}$ and ${ }^{41} \mathrm{~K}$ in the thermal and condensed regime

\begin{tabular}{|c|c|c|c|c|}
\hline \multirow[t]{2}{*}{ Parameter $\backslash$ species } & \multicolumn{2}{|c|}{ Thermal } & \multicolumn{2}{|c|}{$\mathrm{BEC}$} \\
\hline & ${ }^{87} \mathrm{Rb}$ & ${ }^{41} \mathrm{~K}$ & ${ }^{87} \mathrm{Rb}$ & ${ }^{41} \mathrm{~K}$ \\
\hline $\mathrm{T}_{\mathrm{at}}(0) / \mu(0)(\mu \mathrm{K})$ & & & 0.092 & 0.064 \\
\hline $\mathrm{T}_{\mathrm{at}}\left(t_{\mathrm{DKC}}\right) / \mu\left(t_{\mathrm{DKC}}\right)(\mathrm{nK})$ & & & & \\
\hline $\mathrm{t}_{\mathrm{DKC}}(\mathrm{ms})$ & & & 26 & 23 \\
\hline $2 \sigma_{r}(0)(\mathrm{mm})$ & 0.2 & 0.3 & 0.010 & 0.013 \\
\hline $2 \sigma_{r}\left(t_{\mathrm{DKC}}\right)(\mathrm{mm})$ & 1.0 & 1.5 & 0.071 & 0.079 \\
\hline$\sigma_{v}(0)(\mathrm{mm} / \mathrm{s})$ & 14 & 20 & 11 & 14 \\
\hline$\sigma_{v}\left(t_{\mathrm{DKC}}\right)(\mathrm{mm} / \mathrm{s})$ & 2.770 & 4.130 & 0.183 & 0.273 \\
\hline $2 \sigma_{r}\left(t_{\mathrm{DKC}}+0.15 \mathrm{~s}\right)(\mathrm{mm})$ & 1.3 & 2.0 & 0.074 & 0.084 \\
\hline $2 \sigma_{r}\left(t_{\mathrm{DKC}}+0.5 \mathrm{~s}\right)(\mathrm{mm})$ & 3.0 & 4.4 & 0.099 & 0.130 \\
\hline $2 \sigma_{r}\left(t_{\mathrm{DKC}}+10 \mathrm{~s}\right)(\mathrm{mm})$ & 55 & 83 & 1.385 & 2.067 \\
\hline
\end{tabular}

potential $\mu_{\mathrm{BEC}}$ of the $\mathrm{BEC}$ are obtained from

$$
\begin{aligned}
E_{\text {thermal }}(0) & =\frac{3}{2} k_{B} T_{\mathrm{at}}(0) \\
\mu_{\mathrm{BEC}}(0) & =\frac{1}{2} m \omega^{2} R_{\mathrm{TF}}(0)^{2} \\
E_{\text {thermal }}\left(t_{\mathrm{DKC}}\right) & =\frac{3}{2} k_{B} T_{\mathrm{at}}\left(t_{\mathrm{DKC}}\right) \\
E_{\mathrm{BEC}}\left(t_{\mathrm{DKC}}\right) & =\frac{1}{2} m\left(\sigma_{v}\left(t_{\mathrm{DKC}}\right) / \sqrt{7}\right)^{2} .
\end{aligned}
$$

For a better comparison of the two fundamentally different density distributions, the Thomas-Fermi radius $R_{\mathrm{TF}}$ of the isotropic BEC with parabolic density distribution can be related to a Gaussian spatial width $\sigma_{r}$ via $[63]$

$$
R_{\mathrm{TF}}(t)=\sigma_{r}(t) \sqrt{7}
$$

The resulting characteristics of the collimation sequence for ${ }^{87} \mathrm{Rb}$ and ${ }^{41} \mathrm{~K}$ ensembles are given in Table 1 .

The illustrated collimation sequence assumes similar free expansion time $t_{\mathrm{DKC}}$ prior to application of the lens for both regimes. In order to achieve a final expansion behavior of the thermal ensemble similar to the BEC case, $t_{\mathrm{DKC}}$ would need to be significantly increased (about two orders of magnitude, depending on the initial temperature), corresponding to a substantially larger ensemble size at the time of the lens [4]. This is a distinct disadvantage for thermal ensembles, since the DKC technique crucially depends on the harmonicity of the applied lens potential, which has to be verified over the entire spatial extent of the ensemble [11]. Application of velocity-selective pulses for temperature reduction in $1 \mathrm{D}$ has the disadvantage of atom loss [12]. It is therefore not a promising pathway to reach expansion rates for thermal ensembles comparable to those of BECs. Raman sideband cooling [67-69] might be a better alternative for thermal ensembles even if it is limited to about an order of magnitude larger temperatures than what the BEC ensembles could reach. Other recent techniques avoiding evaporation [70-72] might also be promising for a future use in the field.

\subsection{Contrast and detection}

Output states of atom interferometers can be detected by absorption or fluorescence imaging methods. Which method is appropriate depends on the experimental parameters and the information one wants to acquire. One main distinction is whether the relative atom numbers in the output port are counted or if atomic density distributions have to be spatially resolved. Atom number counting is commonly done with fluorescence imaging of the ensembles at the output ports, which relies on the excitation and successive emission of photons by the atoms that are then detected by a simple photo diode or CCD camera. The ensemble has to be excited by a laser beam, which means that it has to have a reasonably compact size to be illuminated. This can usually be achieved with thermal ensembles as well as BECs. The number of atoms contributing constructively to the signal at the output port is given by the product of the excitation probabilities at all interaction times $t_{i}$ :

$$
C\left(t_{n}\right)=p\left(t_{n}\right)=\prod_{i=0}^{n} \mathrm{P}_{\mathrm{exc}}\left(t_{i}\right)
$$

The contrast $\mathrm{C}$ is given by Eq. 25 as the convoluted excitation probability for a given phase-space distribution of the ensemble. Inhomogeneous excitation efficiency or phase gradients e.g., caused by GGs may wash out the contrast [52].

In experiments employing spatial mapping of the output port wave function for the determination of the phase or analysis of features within the atomic ensemble [73], good spatial resolution along with a high signal-tonoise ratio and minimal systematic effects during detection are required. BECs with small spatial spread and expansion rates are thus favored over thermal ensembles to increase the spatial resolution of the CCD picture. Indeed, the high expansion rates of thermal ensembles may at long times lead to densities challenging for absorption imaging due to decreasing signal per volume. 


\section{Comparative performance study}

Based on the discussion of phase shifts and performancelimiting effects in the previous section, we now elaborate on three study cases in which we compare quantum degenerate ensembles to thermal sources. In highly dynamical environments, such as inertial sensing and for navigation purposes, thermal sources may be beneficial since they typically feature more atoms and shorter cycle times, which decreases both, shot noise and integration time. However, a trade-off has to be found for every particular situation due to their relatively high expansion rates and spatial extension. The three specific examples selected for the comparison consist of inertial sensors that could operate beyond state-of-theart in the near future: (i) A ground-based gravimeter with a relative uncertainty of $\Delta g / g=10^{-9}$, (ii) a space gradiometer with a $2.5 \mathrm{mE}$ resolution [74] and (iii) a WEP-test with an uncertainty of $2 \times 10^{-15}$ in the determination of the Eötvös ratio [52]. The interferometer geometries are illustrated in Fig. 2.

In order to evaluate the performance of every regime, we will take typical parameters for thermal ensembles and BECs and assess their performance in each of the three experiments. Details of the ensemble sizes and velocity distributions for both regimes are given in Table 1 at key times of the interferometric sequences. We assume a Gaussian beam of $3 \mathrm{~cm} 1 / e^{2}$-radius with Rabi-frequencies of $\pi /\left(25 \times 10^{-6}\right) \mathrm{Hz}$ (gravimeter and WEP-test) and $\pi /\left(55 \times 10^{-6}\right) \mathrm{Hz}$ (gradiometer) for second order beam-splitting processes, GGs of $10^{-6} \mathrm{~s}^{-2}$ and spurious rotations on the order of $1 \mu \mathrm{rad} / \mathrm{s}$ due to imperfect rotation compensation of the mirror and limited attitude control of the satellite for the two spaceborne missions. The wave front curvature is assumed to be $2.3 \mathrm{~km}$ (gravimeter), $5.6 \mathrm{~km}$ (gradiometer) and $250 \mathrm{~km}$ (WEP-test).

As illustrated in the previous sections, systematic effects linked to GGs and the Coriolis force are connected to the uncertainty of the mean position $\delta_{r}$ and velocity $\delta_{v}$ at the beginning of the interferometry sequence. The number of measurements $\nu_{0}$ required for their characterization (see Eq. 17) is determined for each application such that the largest systematic phase uncertainty related to GGs or rotations (associated with Eqs. 12-16) is below the target uncertainty chosen for the respective measurement.

The number of prerequisite experiments sufficient to suppress the systematic effects below the target uncertainty may differ for the BEC and the thermal case. In our cases, thermal ensembles have three orders of magnitude more atoms and are about 15-20 times larger than BECs after the DKC. Hence, the minimal number of characterization measurements $\nu_{0}$ is reduced by a factor of $2-5$ compared to the BEC case. For the sake of comparability, we choose to compute all systematic effects with $\nu_{0}^{\mathrm{BEC}}$. This enables an evaluation of the performance with a fixed set of parameters.

The systematic phase uncertainty due to WFA is a result of the limited knowledge $\delta R$ about the wave

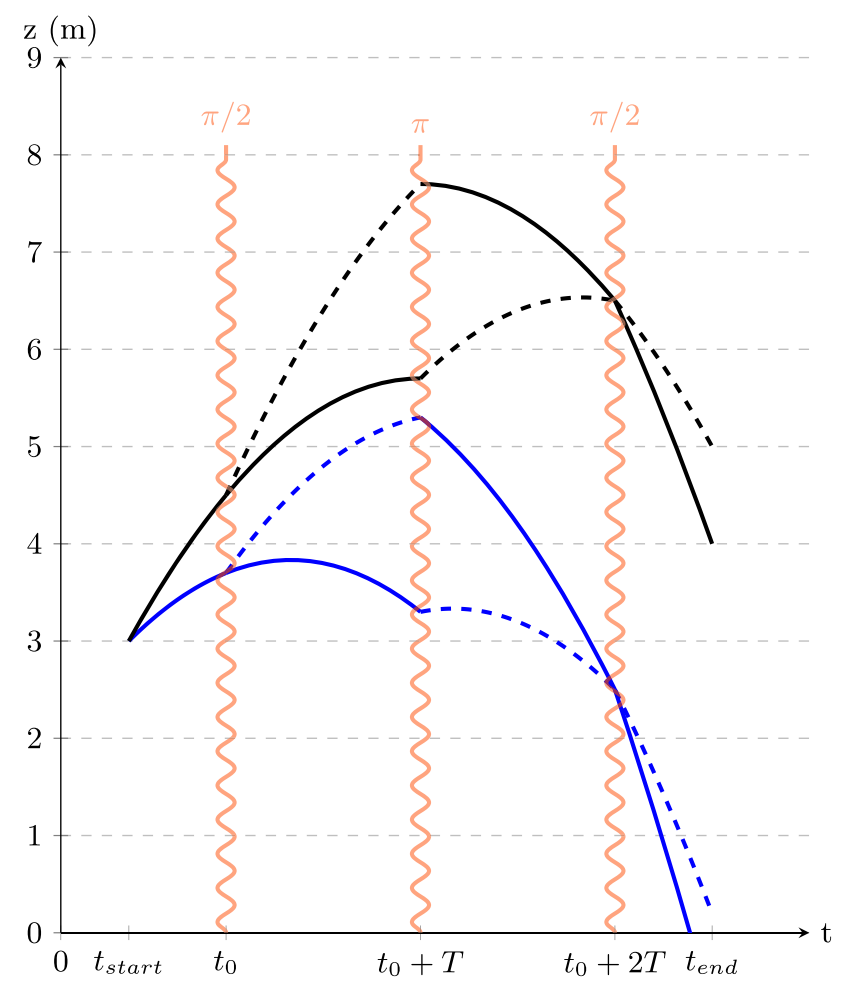

Fig. 2 The geometry for a gradiometer consists of two MZ sequences (black and blue lines) that are operated with an initial separation D at the first beam splitter. Each MZ sequence is formed by three consecutive light pulses that constitute a beam splitter (first $\pi / 2$-pulse), a mirror $(\pi$ pulse) and a merging beam splitter after $2 T$. The dashed lines indicate a change of the internal state due to a momentum transfer by the atom-light interaction. A single MZ sequence corresponds to a gravimeter sequence to measure the coupling constant $g$ of the gravitational field to one species. Two MZ sequences operated with two different species allow for a determination of $\eta$. In this case, the differential velocity at $t=t_{\text {start }}$ of the MZ sequences is set such that they spatially overlap to eliminate the gravity gradient, but remain sensitive to a potentially species specific differential acceleration

front curvature coupling to the effective temperature. To simplify the following considerations, we assume that the wave front curvature $R$ and its uncertainty $\delta R$ are on the same order of magnitude, resulting in Eq. 6 for the assessment of the WFA-induced systematics. In comparison, the statistical uncertainty due to temperature fluctuations is negligible if these fluctuations are assumed to be on the same order of magnitude as the effective temperature of the ensemble.

In order to adapt statistical error contributions such as shot noise and mean-field effects to the desired level of every type of measurement, we calculate the minimum number of iterations $\mathrm{n}_{\text {cycle }}$ until the target uncertainty is reached. The integrated (denoted by subscript i) shot noise is given by

$$
\sigma_{\phi_{\mathrm{SN}}, i}=\sigma_{\phi_{\mathrm{SN}}} / \sqrt{n_{\mathrm{cycle}}}=1 / \sqrt{N_{\mathrm{at}} n_{\mathrm{cycle}}} C
$$


where the contrast $C$ is given by Eq. 25, i.e., the convolved excitation probability. Non-perfect contrast $(C<1)$ increases the shot noise as the number of atoms constituting the statistical sample is reduced.

Mean-field effects contribute a statistical phase uncertainty expressed by Eq. 11 where the ensemble expansion over time is taken into account. This effect integrates down with the number of experiments $n_{\text {cycle }}$ following

$$
\sigma_{\phi_{\mathrm{MF}}, i}=\sigma_{\phi_{\mathrm{MF}}} / \sqrt{n_{\mathrm{cycle}}}
$$

Once the number of cycles is determined by the desired performance, the associated integration time $t_{\text {int }}$ depends on the preparation time $t_{\text {prep }}$ and the interrogation time $2 \mathrm{~T}$ :

$$
\mathrm{t}_{\text {int }}=\left(\mathrm{t}_{\text {prep }}+2 T\right) \mathrm{n}_{\text {cycle }}=\mathrm{t}_{\text {cycle }} \mathrm{n}_{\text {cycle }} .
$$

For a straightforward comparison of the performance differences between BEC and thermal ensemble, the integration time is also chosen to be the same for both regimes, initially determined by the number of cycles needed to suppress the statistical effects of the BEC below the target level. Since thermal sources can be generated within a shorter preparation time, more cycles can be performed during the same integration time according to

$$
n_{\text {cycle }}^{\text {thermal }}=n_{\text {cycle }}^{\mathrm{BEC}} \times t_{\text {cycle }}^{\mathrm{BEC}} / t_{\text {cycle }}^{\text {thermal }} .
$$

In order to estimate the various uncertainties, ensemble properties as spatial and velocity spreads are computed at each atom-light interaction pulse, to take into account the spatial and velocity selectivity of the pulses applying Eqs. 4 and 25. The modified spatial and velocity spreads are the evaluation input for the mean-field effects, the WFA and the estimation of the contrast according to the formulae given in Sect. 2. The results of this study are summarized in Table 2 where the phase uncertainties are normalized as fractional phases $\phi / k_{\text {eff }} g T^{2}$ for the gravimeter and the WEP test. In the gradiometer case, the orders of magnitude are given in units of $\phi / k_{\text {eff }} D T^{2}=\Gamma$ for a baseline D. Here, $\phi_{\text {target }}$ is the upper limit for any systematic or statistical phase uncertainty.

Detailed results for the three science cases are presented in the consecutive sections.

\subsection{Gravimeter}

We start by comparing two ground-based ${ }^{87} \mathrm{Rb}$ gravimeters operated with thermal atoms or BECs, the source characteristics of which are similar to the ones reported in $[5,11,27]$. In both cases, the interrogation time $2 \mathrm{~T}$ equals $150 \mathrm{~ms}$ and the wave front curvature radius is assumed to be $\mathrm{R}=2.3 \mathrm{~km}$.

The first column in Table 2 shows the magnitudes of the different performance-limiting effects discussed in the previous section in units of $\Delta g / g$. The scenario targets an uncertainty of $1 \mu \mathrm{Gal}=10^{-8} \mathrm{~m} / \mathrm{s}^{2}$, corresponding to a fractional phase uncertainty of $\delta_{\phi_{\text {target }}} / k_{\text {eff }} g T^{2}=10^{-9}$.

A thermal ensemble with $10^{9}{ }^{87} \mathrm{Rb}$ atoms would have a diameter of $2 \sigma_{r}\left(t_{\mathrm{DKC}}\right)=1 \mathrm{~mm}$ after the DKC pulse. The velocity spread at the lens is $2.7 \mathrm{~mm} / \mathrm{s}$. The convolved excitation efficiency at the last beam splitter is $57 \%$.

Adopting a BEC source as in [11], it is reasonable to assume a collimation of the ensemble to an effective temperature of $50 \mathrm{pK}$ for $10^{6}$ atoms. The convolved excitation efficiency at the last beam splitter is $99 \%$ for an ensemble diameter of $2 R_{\mathrm{TF}}\left(t_{\mathrm{DKC}}\right)=0.19 \mathrm{~mm}$ and a velocity spread of $183 \mu \mathrm{m} / \mathrm{s}$. Although the order of magnitude of the initial atom number is three times larger for the thermal atoms, the shot noise is only one order of magnitude below the one of the BEC due to the reduced contrast.

Theoretically, the target uncertainty of $\Delta g / g=10^{-9}$ is reached in both cases after only one verification shot and integration over seven (thermal, full cycle time $0.48 \mathrm{~s}$ ) or three (BEC, full cycle time $1.15 \mathrm{~s}$ ) experimental cycles corresponding to a few seconds of integration time. All statistical and systematic effects are below the target uncertainty hinting toward the possibility to use either of the source concepts.

However, if a better performance of the gravimeter is sought for, the first limit to tackle would be the mean-field effects at $9.2 \times 10^{-10}$ in the BEC case and wave front distortions at $3.4 \times 10^{-10}$ in the thermal one. Mean-field effects are treated here as a statistical phenomenon, hence one can integrate down the phase uncertainty for the BEC case by increasing the number of experiments.

As mentioned, WFA are not a statistical but a systematic phenomenon and cannot be integrated down by adding verification shots. Therefore thermal sources are limited by WFA to the $10^{-10}$ level, whereas the BECs could improve on the accuracy up to the $10^{-13}$ level.

\subsection{Gradiometer}

Here, we are address a satellite gradiometer as proposed in [74]. It features a baseline of $D=0.5 \mathrm{~m}$ separating the two interferometers, an interrogation time of $2 \mathrm{~T}=10 \mathrm{~s}$ (full cycle time of $20 \mathrm{~s}$ ) and a targeted uncertainty of $2.5 \mathrm{mE}$, clearly beyond the current state of the art. We adapt the interferometry time from $2 \mathrm{~T}=10 \mathrm{~s}$ to $2 \mathrm{~T}=0.5 \mathrm{~s}$ to constrain the ensemble size at a detectable level in the thermal case.

The center column of Table 2 displays the order of magnitude of uncertainties in the gradient determination related to the different effects. Here, the numbers are given as spurious gradients in units of $\Gamma$ by dividing each phase uncertainty by $k_{\text {eff }} D T^{2}$.

The phase uncertainties due to GGs, the Coriolis force and mean-field effects receive contributions from both individual interferometers. Through Eq. 17, the initial spatial and velocity spreads $\sigma_{r, v ; 1,2}$ of interferometer 1,2 enter the systematic uncertainties given in 
Table 2 Estimation of statistical and systematic uncertainties for three scenarios: a lab-based ${ }^{87} \mathrm{Rb}$ gravimeter ([5], Sect. 3.1), a space-borne ${ }^{87} \mathrm{Rb}$ gradiometer ([74], Sect. 3.2) and a satellite ${ }^{87} \mathrm{Rb} /{ }^{41} \mathrm{~K}$ WEP-test analogous to the STEQUEST mission ([52], Sect. 3.3)

\begin{tabular}{|c|c|c|c|c|c|c|}
\hline \multirow[t]{2}{*}{ Parameter $\backslash$ case } & \multicolumn{2}{|c|}{ Gravimeter } & \multicolumn{2}{|c|}{ Gradiometer } & \multicolumn{2}{|c|}{ WEP-test } \\
\hline & Thermal & $\mathrm{BEC}$ & Thermal & $\mathrm{BEC}$ & Thermal & $\mathrm{BEC}$ \\
\hline $\mathrm{N}_{\text {at }}$ (initial) & $1 \times 10^{9}$ & $1 \times 10^{6}$ & $1 \times 10^{9}$ & $1 \times 10^{6}$ & $1 \times 10^{9}$ & $1 \times 10^{6}$ \\
\hline $\mathrm{T}_{\mathrm{at}}(\mathrm{K})$ & $80 \times 10^{-9}$ & $50 \times 10^{-12}$ & $80 \times 10^{-9}$ & $50 \times 10^{-12}$ & $80 \times 10^{-9}$ & $50 \times 10^{-12}$ \\
\hline $\mathrm{P}_{\text {exc }}$ & 0.57 & 0.99 & 0.33 & 0.97 & 0.43 & 0.99 \\
\hline $\mathrm{t}_{\text {int }}(\mathrm{s}) ; \mathrm{n}_{\text {cycle }}$ & $3.36 ; 7$ & $3.45 ; 3$ & $86400 ; 72000$ & $86400 ; 4320$ & $2 \times 10^{7} ; 2.4 \times 10^{7}$ & $2 \times 10^{7} ; 10^{6}$ \\
\hline$\nu_{0}$ & \multicolumn{2}{|l|}{ 等 } & \multicolumn{2}{|c|}{50} & \multicolumn{2}{|l|}{$10^{6}$} \\
\hline $2 \mathrm{~T}(\mathrm{~s})$ & \multicolumn{2}{|c|}{$150 \times 10^{-3}$} & 0.5 & 10 & 0.5 & 10 \\
\hline $\mathcal{O}\left(\delta_{\phi_{\text {target }}}\right)$ & $\Delta g / g$ & $=10^{-9}$ & \multicolumn{2}{|c|}{$\Gamma=2.5 \mathrm{mE}=2.5 \times 10^{-12} \mathrm{~s}^{-2}$} & \multicolumn{2}{|c|}{$\eta=2 \times 10^{-15}$} \\
\hline $\mathcal{O}\left(\sigma_{\phi_{\mathrm{SN}}}\right)$ & $1.2 \times 10^{-11}$ & $3.3 \times 10^{-10}$ & $5.0 \times 10^{-13} \mathrm{~s}^{-2}$ & $5.5 \times 10^{-14} \mathrm{~s}^{-2}$ & $1.2 \times 10^{-15}$ & $2.1 \times 10^{-16}$ \\
\hline $\mathcal{O}\left(\delta_{\phi_{\mathrm{GG}}}\right)$ & $4.9 \times 10^{-15}$ & $1.1 \times 10^{-14}$ & $2.4 \times 10^{-14} \mathrm{~s}^{-2}$ & $9.9 \times 10^{-13} \mathrm{~s}^{-2}$ & $1.1 \times 10^{-17}$ & $4.8 \times 10^{-16}$ \\
\hline $\mathcal{O}\left(\delta_{\phi_{\mathrm{C}}}\right)$ & $1.8 \times 10^{-14}$ & $3.7 \times 10^{-14}$ & $7.0 \times 10^{-14} \mathrm{~s}^{-2}$ & $1.5 \times 10^{-13} \mathrm{~s}^{-2}$ & $3.7 \times 10^{-17}$ & $7.6 \times 10^{-17}$ \\
\hline $\mathcal{O}\left(\delta_{\phi_{\mathrm{WFA}}}\right)$ & $3.4 \times 10^{-10}$ & $2.1 \times 10^{-13}$ & $1.0 \times 10^{-12} \mathrm{~s}^{-2}$ & $4.4 \times 10^{-15} \mathrm{~s}^{-2}$ & $1.3 \times 10^{-12}$ & $8.0 \times 10^{-16}$ \\
\hline $\mathcal{O}\left(\sigma_{\phi_{\mathrm{MF}}}\right)$ & $1.3 \times 10^{-11}$ & $9.2 \times 10^{-10}$ & $4.7 \times 10^{-13} \mathrm{~s}^{-2}$ & $5.4 \times 10^{-13} \mathrm{~s}^{-2}$ & $1.1 \times 10^{-15}$ & $1.8 \times 10^{-15}$ \\
\hline
\end{tabular}

The phase uncertainties are given as fractions $\Delta a / g=\delta_{\phi} / k_{\text {eff }} g T^{2}$ (gravimeter and WEP-test) and $\Delta \Gamma=\delta_{\phi} / k_{\text {eff }} D T^{2}($ space gradiometer). The expansion sequence over the course of the atom interferometer is calculated in Table 1 . Systematic effects are denoted by $\delta$, while statistical effects are denoted by $\sigma$ and calculated after integrating over a number $n_{\text {cycle }}$ of experiments with $\mathrm{N}_{\text {at }}$ atoms in each cycle. Gravity gradients are abbreviated with GG, the Coriolis effect with C, wave front aberrations by WFA, shot noise with SN and mean-field effects by MF

Eqs. 12 and 16 as

$$
\sigma_{r, v}=\sqrt{\sigma_{r, v ; 1}^{2}+\sigma_{r, v ; 2}^{2}}
$$

supposing uncorrelated source noise. For initial spreads with the same width $\sigma_{r, v ; 1}=\sigma_{r, v ; 2}$, this yields a factor of $\sqrt{2}$, which results in an integration behavior during the characterization measurements of

$$
\delta_{\phi, i}=\delta_{\phi} \sqrt{2} / \sqrt{\nu_{0}}
$$

in the case of GGs and Coriolis effect. The same holds true for the integrated shot noise, which is increased by a factor of $\sqrt{2}$ compared to Eq. 26:

$$
\sigma_{\phi_{\mathrm{SN}}, i}=\sqrt{2} / \sqrt{N_{\mathrm{at}} n_{\mathrm{cycle}}} C
$$

and for the mean-field effects, which are uncorrelated between the two branches of the interferometer:

$$
\sigma_{\phi_{\mathrm{MF}}, i}=\sqrt{2} \sigma_{\phi_{\mathrm{MF}}} / \sqrt{n_{\mathrm{cycle}}}
$$

Interestingly, following our treatment in Sect. 2.3, we find that due to the different expansion behavior and a higher atom number, the mean-field effects of the thermal ensemble are comparable, in magnitude, to that of the BEC on the time scales we are investigating (see Table 2 and Fig. 3).

Assuming the same velocity spread for the two ensembles in the two interferometers and - as for the gravimeter case - the simplification of a constant curvature, the differential phase shift vanishes. Here, we drop this simplification and consider the propagation of a Gaussian beam which leads to a local dependency of the curvature and thus to a nonvanishing phase shift in the differential signal. Assuming a residual radius of curvature of the retro-reflection mirror of $4 \mathrm{~km}$ and a propagating the laser beam as outlined in [74] introduces the differential phase shift as reported in Table 2.

To reach the uncertainty goal of $2.5 \mathrm{mE}$, an integration time on the order of 1 day is required in both cases. Albeit the high expansion rate of the thermal ensemble is accounted for with a shorter interrogation time of $0.5 \mathrm{~s}$ (full cycle time of $1.2 \mathrm{~s}$ ), the shot noise uncertainty in the thermal case exceeds the one of the BEC case as the contrast drops to $33 \%$ (97\% for the BEC after $10 \mathrm{~s})$. Again, the WFA would hinder any further attempts for a better performance below the $10^{-12} \mathrm{~s}^{-2}$ level. All other systematic effects are complying with the performance required from this sensor and can be reduced by an increased number of verification shots.

Being limited by WFA at the level of $4.4 \times 10^{-15}$, the BEC clearly leaves more room for improvement compared to thermal sources, which are bound three orders of magnitude above. Therefore, using a BEC source in this scenario is advantageous since it does neither suffer from a worse integration time nor a larger mean-field effect, yet significantly extends the achievable accuracy compared to the thermal source.

\subsection{WEP-test}

The WEP-test example is based on the parameters of the STE-QUEST satellite mission proposed in [52]. We here assume the test pair ${ }^{41} \mathrm{~K}$ and ${ }^{87} \mathrm{Rb}$. It aims at an Eötvös ratio $\eta$ determined with an uncertainty of $2 \times 10^{-15}$. As for the gradiometer, we compare a thermal 


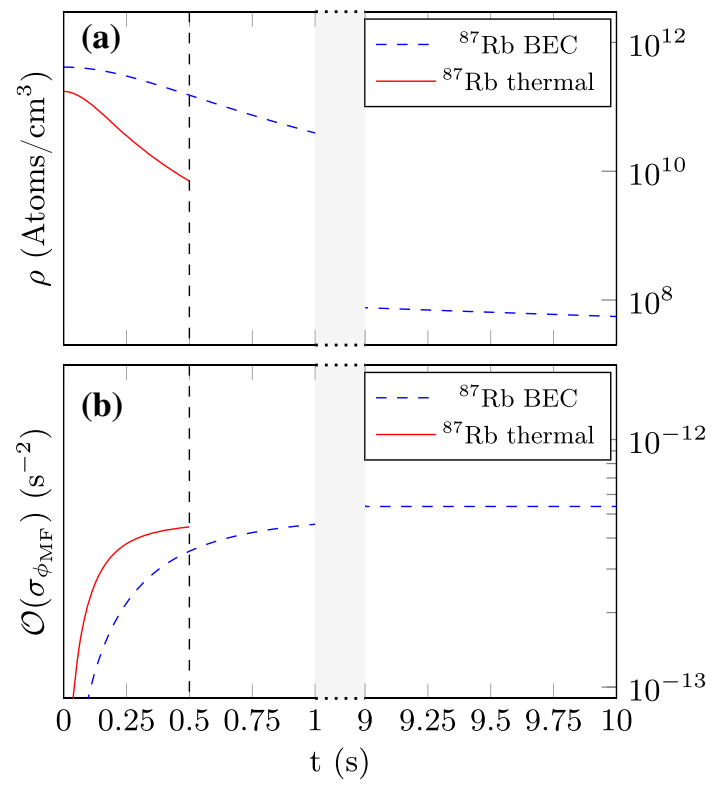

Fig. 3 Atomic densities and time averaged mean-field statistical uncertainty for the space-gradiometer scenario described in Table 2. a Time-dependent density $\rho$ of the ensembles during the interferometer time based on the DKC sequence described in Table 1 . b Fractional statistical phase uncertainty $\mathcal{O}\left(\sigma_{\phi_{\mathrm{MF}}}\right)$ due to mean-field effects according to Eq. 11 integrated over a number of $n_{\text {cycle }}$ experiments

ensemble at $0.5 \mathrm{~s}$ of interrogation time (full cycle time of $0.83 \mathrm{~s}$ ) with a BEC at $10 \mathrm{~s}$ (full cycle time of $20 \mathrm{~s}$ ) of interrogation time to ensure nonvanishing contrast and technically manageable ensemble sizes in both cases. The interrogation time $2 \mathrm{~T}$ is the same for both species in the respective scenario. In the last column of Table 2 , the results of the comparison are displayed. The wave front curvature radius is assumed to be controlled at the level of $R=250 \mathrm{~km}$.

For the determination of the systematic effects one has to take into account the differences of the two atomic species such as the different expansion rates, atomic masses and initial conditions like spatial spread. The two different species-specific excitation rates are also evaluated and the minimum given in Table 2.

The effects of GGs and Coriolis force do not scale with $\sqrt{2}$ in this case, but rather with the mean square of two uncorrelated spreads (see Eq. 30). With two different species propagating in the arms, the meanfield effects are calculated as the mean square sum of the individual mean-field effects of ${ }^{87} \mathrm{Rb}$ and ${ }^{41} \mathrm{~K}$ as in Eq. 11. As for the gradiometer, one benefits from the differential suppression of WFA when matching the expansion rates of the ensembles [52]. As two different sources and two different species are used for the production of the ensembles, the systematic differential phase uncertainty is given by

$$
\delta_{\phi \mathrm{WFA}}=\frac{k_{\mathrm{eff}}}{R} k_{B}\left(\frac{T_{\mathrm{at}, \mathrm{K}}}{m_{\mathrm{at}, \mathrm{K}}}-\frac{T_{\mathrm{at}, \mathrm{Rb}}}{m_{\mathrm{at}, \mathrm{Rb}}}\right) T^{2},
$$

analogous to Eq. 6. By experimentally matching the velocity spreads of the two ensembles to the $20 \%$ level in both arms, the WFA are suppressed by a factor of 3 .

In the BEC case and in order for the statistical effects to be consistent with the mission performance goal, $10^{6}$ experimental cycles $n_{\text {cycle }}$ are needed. This leads to a full mission time on the order of five years in case of a highly elliptical orbit operation. To reach the targeted uncertainty, an additional $10^{6}$ verification measurements $\nu_{0}$ are necessary. These measurements are included in the total mission time as they are performed on the transition between perigee and apogee parts of the orbit not dedicated to science measurements [52]. The contrast at the end of the sequence is at $99 \%$ for both, ${ }^{87} \mathrm{Rb}$ and ${ }^{41} \mathrm{~K}$, and for the chosen set of parameters it is feasible to achieve the missions goals with condensed ensembles.

With a thermal ensemble, one would need an integration time on the order of $10^{8} \mathrm{~s}$ to suppress mean-field effects, and $10^{6}$ verification shots to estimate the phase uncertainty due to GGs and the Coriolis effect to a sufficient level. Moreover, even for the reduced interrogation time of $0.5 \mathrm{~s}$, the contrast is at $43 \%$ and the shot noise is almost 6 times larger than in the BEC case.

We conclude that - for the chosen set of parametersit is possible to achieve the mission goals with condensed ensembles. The thermal case is, however, limited to the $10^{-12}$ level due to the WFA effects stemming from the large expansion rates of the thermal ensembles [44]. In order to reach a reduced velocity spread with a thermal ensemble comparable to that of a BEC, the DKC would be required to handle ensembles with a diameter on the order of $0.5 \mathrm{~m}$ after a pre-lens free expansion time of several seconds. As for the spacegradiometer, this is unpractical for manipulation and excitation reasons: The required beam quality in terms of WFA over several decimeters in diameter and high laser power to achieve reasonable excitation rates pose a considerable technical challenge. In the condensed regime, a simultaneous collimation of the dual-source was recently considered in reference [75] and shown to be feasible.

\section{Discussion and conclusion}

In this paper, the current limits for state-of-the-art precision experiments with atom interferometry were analyzed. A particular emphasis was put on the comparison of the statistical and systematic uncertainties between condensed and thermal ensembles. Three detailed study cases of a laboratory-based gravimeter, a space gradiometer and a satellite WEP-test were chosen to illustrate the limits of each regime.

Thermal sources benefit from a shorter cycle time, smaller mean-field effects and larger atom numbers compatible with experiments where moderate scale factors suffice or rapid readouts are required (gravimetry scenario). This is, however, beneficial at short interferometry times only. When moving beyond the current 
state of the art, i.e., from drift times of a fraction of a second to a few seconds, this advantage is lost. In our last two study cases, the scenarios utilizing BECs show the same level of shot noise and magnitude of mean-field effects. Moreover, the condensed sources benefit from a very large contrast (close to 1) when compared to their thermal counterparts. More dramatically, the WFA set an ultimate limit for thermal ensembles that would not be compatible with long interrogation times, which are required for advanced scenarios. For BEC ensembles, their compact sizes make this limit at least three orders of magnitude lower, highlighting their potential in the field of metrology.

Small-scale distortions (few $\mu m$ ) of the optical beams [76], not considered in this article, can hint to a disadvantage for the BEC samples by means of averaging effects for WFA. However, the flexibility in tuning their initial size [75] mitigates this effect and could bring them to starting sizes similar to thermal ensembles if necessary. This engineering of the BEC size allows for a distinct analysis of WFA with long and short periodicity $[5,44]$. This might be especially relevant on short time scales, i.e., for very small ensemble sizes. Their subsequent expansion could still be limited to a few $\mathrm{mm}$ thanks to the DKC technique. In consequence, a trade-off between the size-stretch-induced phase uncertainties, e.g., to balance the level of GGs or Coriolis systematics versus WFA effects is required. This appears to be feasible, especially if one considers gravity gradient compensation schemes as the one in $[55,74,77]$.

Other considerations that are not reflected by our study would further consolidate the BEC choice. Indeed, we optimistically assumed here that thermal ensembles can be collimated to the $80 \mathrm{nK}$ level and that the same level of efficiency in preparing, transporting and engineering of their the quantum states can be achieved as for BECs. As a conclusion, thermal and BEC sources could equally be employed in relatively short interferometry times (a few hundred $\mathrm{ms}$ ) for the same performance. With respect to longer times, BEC sources are clearly more advantageous since size-related systematic effects are several orders of magnitude smaller than those of thermal ensembles.

Acknowledgements The authors would like to thank Dennis Schlippert and Waldemar Herr for constructive criticism of the manuscript. This work is supported by the German Space Agency (DLR) with funds provided by the Federal Ministry for Economic Affairs and Energy (BMWi) due to an enactment of the German Bundestag under Grant Nos. 50WM1861 (CAL) and 50WM-2060 (CARIOQA), 50RK1957 (QGYRO), 50WM1952 (QUANTUS-VFallturm), and 50WP1700 (BECCAL), by "Nieders "achsisches Vorab" through the "Quantum- and Nano-Metrology (QUANOMET)" initiative within the project QT3 funded by the Deutsche For-schungs-ge-mein-schaft (DFG, German Research Foundation) under Germany's Excellence Strategy - EXC 2123 QuantumFrontiers, - 390837967 and under the CRC 1227 (DQmat) within Projects No. A05, No. B07 and No. B09 and through "Förderung von Wissenschaft und Technik in For-schung und Lehre" for the initial fund- ing of research in the new DLR Institute (DLR-SI). We also acknowledge support by the QUEST-LFS, the Verein Deutscher Ingenieure (VDI) with funds provided by the Federal Ministry of Education and Research (BMBF) under Grant No. VDI 13N14838 (TAIOL). SL acknowledges the support of the IP@Leibniz program of the Leibniz University of Hanover for travel grants supporting his stays in France.

\section{Author contributions}

All the authors were involved in the preparation of the manuscript. All the authors have read and approved the final manuscript.

Data Availability Statement This manuscript has no associated data or the data will not be deposited. [Authors' comment: This is theoretical study and no experimental data has been listed.]

Funding Open Access funding enabled and organized by Projekt DEAL.

Open Access This article is licensed under a Creative Commons Attribution 4.0 International License, which permits use, sharing, adaptation, distribution and reproduction in any medium or format, as long as you give appropriate credit to the original author(s) and the source, provide a link to the Creative Commons licence, and indicate if changes were made. The images or other third party material in this article are included in the article's Creative Commons licence, unless indicated otherwise in a credit line to the material. If material is not included in the article's Creative Commons licence and your intended use is not permitted by statutory regulation or exceeds the permitted use, you will need to obtain permission directly from the copyright holder. To view a copy of this licence, visit http://creativecomm ons.org/licenses/by/4.0/.

\section{References}

1. R. Geiger, A. Landragin, S. Merlet, F. Pereira Dos Santos, High-accuracy inertial measurements with coldatom sensors (2020). arXiv:2003.12516

2. R. Bouchendira, P. Cladé, S. Guellati-Khélifa, F. Nez, F. Biraben, Phys. Rev. Lett. 106, 080801 (2011)

3. P. Asenbaum, C. Overstreet, M. Kim, J. Curti, M.A. Kasevich, Phys. Rev. Lett. p. 5 (2020)

4. S. Loriani, D. Schlippert, C. Schubert, S. Abend, H. Ahlers, W. Ertmer, J. Rudolph, J.M. Hogan, M.A. Kasevich, E.M. Rasel et al., New J. Phys. 21, 063030 (2019)

5. A. Louchet-Chauvet, T. Farah, Q. Bodart, A. Clairon, A. Landragin, S. Merlet, F. Pereira Dos Santos, New J. Phys. 13, 065025 (2011)

6. V. Schkolnik, B. Leykauf, M. Hauth, C. Freier, A. Peters, Appl. Phys. B 120, 311 (2015)

7. S. Chu, J.E. Bjorkholm, A. Ashkin, J.P. Gordon, L.W. Hollberg, Opt. Lett. 11, 73 (1986) 
8. H. Ammann, N. Christensen, Phys. Rev. Lett. 78, 2088 (1997)

9. H. Müntinga, H. Ahlers, M. Krutzik, A. Wenzlawski, S. Arnold, D. Becker, K. Bongs, H. Dittus, H. Duncker, N. Gaaloul et al., Phys. Rev. Lett. 110, 093602 (2013)

10. T. Kovachy, J.M. Hogan, A. Sugarbaker, S.M. Dickerson, C. Donnelly, C. Overstreet, M.A. Kasevich, Phys. Rev. Lett. 114, 143004 (2015)

11. J. Rudolph, W. Herr, C. Grzeschik, T. Sternke, A. Grote, M. Popp, D. Becker, H. Müntinga, H. Ahlers, A. Peters et al., New J. Phys. 17, 065001 (2015)

12. M. Kasevich, D.S. Weiss, E. Riis, K. Moler, S. Kasapi, S. Chu, Phys. Rev. Lett. 66, 2297 (1991)

13. A. Peters, K.Y. Chung, S. Chu, Nature 400, 849 (1999)

14. T.L. Gustavson, P. Bouyer, M.A. Kasevich, Phys. Rev. Lett. 78, 2046 (1997)

15. T.L. Gustavson, A. Landragin, M.A. Kasevich, Class. Quantum Gravity 17, 2385 (2000)

16. D.S. Durfee, Y.K. Shaham, M.A. Kasevich, Phys. Rev. Lett. 97, 240801 (2006)

17. B. Canuel, F. Leduc, D. Holleville, A. Gauguet, J. Fils, A. Virdis, A. Clairon, N. Dimarcq, C.J. Bordé, A. Landragin et al., Phys. Rev. Lett. 97, 010402 (2006)

18. I. Dutta, D. Savoie, B. Fang, B. Venon, C.L.G. Alzar, R. Geiger, A. Landragin, Phys. Rev. Lett. 116, 183003 (2016)

19. M. Snadden, J. McGuirk, P. Bouyer, K. Haritos, M. Kasevich, Phys. Rev. Lett. 81, 971 (1998)

20. A. Bertoldi, G. Lamporesi, L. Cacciapuoti, M. de Angelis, M. Fattori, T. Petelski, A. Peters, M. Prevedelli, J. Stuhler, G.M. Tino, Eur. Phys. J. D Atom. Mol. Opt. Plasma Phys. 40, 271 (2006)

21. R.H. Parker, C. Yu, W. Zhong, B. Estey, H. Müller, Science 360, 191 (2018)

22. G. Rosi, F. Sorrentino, L. Cacciapuoti, M. Prevedelli, G.M. Tino, Nature 510, 518 (2014)

23. S. Fray, C.A. Diez, T.W. Hänsch, M. Weitz, Phys. Rev. Lett. 93, 240404 (2004)

24. D. Schlippert, J. Hartwig, H. Albers, L.L. Richardson, C. Schubert, A. Roura, W.P. Schleich, W. Ertmer, E.M. Rasel, Phys. Rev. Lett. 112, 203002 (2014)

25. M.G. Tarallo, T. Mazzoni, N. Poli, D.V. Sutyrin, X. Zhang, G.M. Tino, Phys. Rev. Lett. 113, 023005 (2014)

26. L. Zhou, S. Long, B. Tang, X. Chen, F. Gao, W. Peng, W. Duan, J. Zhong, Z. Xiong, J. Wang et al., Phys. Rev. Lett. 115, 013004 (2015)

27. H. Albers, A. Herbst, L.L. Richardson, H. Heine, D. Nath, J. Hartwig, C. Schubert, C. Vogt, M. Woltmann, C. Lämmerzahl et al., Eur. Phys. J. D 74, 1-9 (2020)

28. P.W. Graham, J.M. Hogan, M.A. Kasevich, S. Rajendran, Phys. Rev. Lett. 110, 171102 (2013)

29. J.M. Hogan, M.A. Kasevich, Phys. Rev. A 94, 104022 (2016)

30. C. Schubert, D. Schlippert, S. Abend, E. Giese, A. Roura, W.P. Schleich, W. Ertmer, E.M. Rasel, Scalable, symmetric atom interferometer for infrasound gravitational wave detection (2019). arXiv:1909.01951

31. B. Canuel, A. Bertoldi, L. Amand, E.P. di Borgo, T. Chantrait, C. Danquigny, M.D. Álvarez, B. Fang, A. Freise, R. Geiger et al., Sci. Rep. 8, 1-23 (2018)

32. G.M. Tino, Testing Gravity with Cold Atom Interferometry: Results and Prospects, vol. 2009, p. 01484 (2020)
33. K. Bongs, M. Holynski, J. Vovrosh, P. Bouyer, G. Condon, E. Rasel, C. Schubert, W.P. Schleich, A. Roura, Nat. Rev. Phys. 1, 731 (2019)

34. V. Ménoret, P. Vermeulen, N.L. Moigne, S. Bonvalot, P. Bouyer, A. Landragin, B. Desruelle, Sci. Rep. 8 (2018)

35. J.L. Gouet, T. Mehlstäubler, J. Kim, S. Merlet, A. Clairon, A. Landragin, F. Pereira Dos Santos, Appl. Phys. B 92, 133 (2008)

36. Z.K. Hu, B.L. Sun, X.C. Duan, M.K. Zhou, L.L. Chen, S. Zhan, Q.Z. Zhang, J. Luo, Phys. Rev. A 88, 043610 (2013)

37. P. Gillot, B. Cheng, A. Imanaliev, S. Merlet, F. Pereira Dos Santos, The LNE-SYRTE cold atom gravimeter, in 2016 European Frequency and Time Forum (EFTF) (IEEE, 2016)

38. C. Freier, M. Hauth, V. Schkolnik, B. Leykauf, M. Schilling, H. Wziontek, H.G. Scherneck, J. Müller, A. Peters, J. Phys. Conf. Ser. 723, 012050 (2016)

39. P. Berg, S. Abend, G. Tackmann, C. Schubert, E. Giese, W.P. Schleich, F.A. Narducci, W. Ertmer, E.M. Rasel, Phys. Rev. Lett. 114, 063002 (2015)

40. J. Stockton, K. Takase, M.A. Kasevich, Phys. Rev. Lett. 107, 133001 (2011)

41. D. Savoie, M. Altorio, B. Fang, L.A. Sidorenkov, R. Geiger, A. Landragin, Sci. Adv. 4, eaau7948 (2018)

42. K.S. Hardman, P.J. Everitt, G.D. McDonald, P. Manju, P.B. Wigley, M.A. Sooriyabandara, C.C.N. Kuhn, J.E. Debs, J.D. Close, N.P. Robins, Phys. Rev. Lett. 117, $138501(2016)$

43. J.E. Debs, P.A. Altin, T.H. Barter, D. Döring, G.R. Dennis, G. McDonald, R.P. Anderson, J.D. Close, N.P. Robins, Phys. Rev. A 84, 033610 (2011)

44. R. Karcher, A. Imanaliev, S. Merlet, F. Pereira Dos Santos, New J. Phys. 20, 113041 (2018)

45. S. Abend, M. Gebbe, M. Gersemann, H. Ahlers, H. Müntinga, E. Giese, N. Gaaloul, C. Schubert, C. Lämmerzahl, W. Ertmer et al., Phys. Rev. Lett. 117, 203003 (2016)

46. N. Heine, J. Matthias, M. Sahelgozin, W. Herr, S. Abend, L. Timmen, J. Müller, E.M. Rasel, Eur. Phys. J. 74, $174(2020)$

47. S.M. Dickerson, J.M. Hogan, A. Sugarbaker, D.M.S. Johnson, M.A. Kasevich, Phys. Rev. Lett. 111, 083001 (2013)

48. C. Yu, W. Zhong, B. Estey, J. Kwan, R.H. Parker, H. Müller, Ann. Phys. 531, 1800346 (2019)

49. P. Cladé, F. Nez, F. Biraben, S. Guellati-Khelifa, C.R. Phys. 20, 77 (2019)

50. L. Morel, Z. Yao, P. Cladé, S. Guellati-Khélifa, Nature 588, $61(2020)$

51. A.O. Jamison, B. Plotkin-Swing, S. Gupta, Phys. Rev. A 90, 063606 (2014)

52. D.N. Aguilera, H. Ahlers, B. Battelier, A. Bawamia, A. Bertoldi, R. Bondarescu, K. Bongs, P. Bouyer, C. Braxmaier, L. Cacciapuoti et al., Class. Quantum Gravity 31, 115010 (2014)

53. B. Battelier, J. Bergé, A. Bertoldi, L. Blanchet, K. Bongs, P. Bouyer, C. Braxmaier, D. Calonico, P. Fayet, N. Gaaloul et al. (2019). arXiv:1908.11785

54. J. Williams, S.W. Chiow, N. Yu, H. Müller, New J. Phys. 18, $025018(2016)$ 
55. S. Loriani, C. Schubert, D. Schlippert, W. Ertmer, F. Pereira Dos Santos, E.M. Rasel, N. Gaaloul, P. Wolf, Phys. Rev. D 102, 124043 (2020)

56. M. Gebbe, S. Abend, J. Siemß, M. Gersemann, H. Ahlers, H. Müntinga, S. Herrmann, N. Gaaloul, C. Schubert, K. Hammerer et al., Twin-lattice atom interferometry (2019). arXiv:1907.08416

57. P. Cheinet, Ph.D. thesis, Universiteé Paris (2006). https://tel.archives-ouvertes.fr/tel-00070861v1

58. D. Guéry-Odelin, Phys. Rev. A 66, 033613 (2002)

59. C. Brif, B.P. Ruzic, G.W. Biedermann, Characterization of errors in interferometry with entangled atoms (2020). arXiv:2007.03306

60. J.M. Hogan, D.M.S. Johnson, M.A. Kasevich, Proceedings of the International School of Physics Enrico Fermi (Societa Italiana di Fisica and IOS Press, Amsterdam, 2009), pp. 411-447

61. T. Müller, M. Gilowski, M. Zaiser, P. Berg, C. Schubert, T. Wendrich, W. Ertmer, E.M. Rasel, Eur. Phys. J. D 53, 273 (2009)

62. C. Antoine, C.J. Borde, J. Opt. B: Quantum Semiclassical Opt. 5, S199 (2003)

63. R. Corgier, S. Amri, W. Herr, H. Ahlers, J. Rudolph, D. Guery-Odelin, E.M. Rasel, E. Charron, N. Gaaloul, New J. Phys. 20, 055002 (2018)

64. Y. Castin, R. Dum, Phys. Rev. Lett. 77, 5315 (1996)

65. Y. Kagan, E.L. Surkov, G.V. Shlyapnikov, Phys. Rev. A 54, R1753 (1996)

66. P. Pedri, D. Guéry-Odelin, S. Stringari, Phys. Rev. A 68, 043608 (2003)

67. S.E. Hamann, D.L. Haycock, G. Klose, P.H. Pax, I.H. Deutsch, P.S. Jessen, Phys. Rev. Lett. 80, 4149 (1998)
68. V. Vuletić, C. Chin, A.J. Kerman, S. Chu, Phys. Rev. Lett. 81, 5768 (1998)

69. B. Estey, C. Yu, H. Müller, P.C. Kuan, S.Y. Lan, Phys. Rev. Lett. 115, 083002 (2015)

70. S. Stellmer, B. Pasquiou, R. Grimm, F. Schreck, Phys. Rev. Lett. 110, 263003 (2013)

71. A. Urvoy, Z. Vendeiro, J. Ramette, A. Adiyatullin, V. Vuletić, Phys. Rev. Lett. 122, 203202 (2019)

72. D.S. Naik, H. Eneriz-Imaz, M. Carey, T. Freegarde, F. Minardi, B. Battelier, P. Bouyer, A. Bertoldi, Phys. Rev. Res. 2, 013212 (2020)

73. A. Sugarbaker, S.M. Dickerson, J.M. Hogan, D.M.S. Johnson, M.A. Kasevich, Phys. Rev. Lett. 111, 083001 (2013)

74. A. Trimeche, B. Battelier, D. Becker, A. Bertoldi, P. Bouyer, C. Braxmaier, E. Charron, R. Corgier, M. Cornelius, K. Douch et al., Class. Quantum Gravity 36, 215004 (2019)

75. R. Corgier, S. Loriani, H. Ahlers, K. Posso-Trujillo, C. Schubert, E.M. Rasel, E. Charron, N. Gaaloul, New J. Phys. 22, 123008 (2020)

76. S. Bade, L. Djadaojee, M. Andia, P. Cladé, S. GuellatiKhelifa, Phys. Rev. Lett. 121, 073603 (2018)

77. A. Roura, Phys. Rev. Lett. 118, 160401 (2017) 